\title{
Short Communication: Immune Activation Is Present in HIV-1-Exposed Seronegative Individuals and Is Independent of Microbial Translocation
}

\author{
Irma Saulle,' Mara Biasin,, Federica Gnudi,, Veronica Rainone,' Salomè Valentina Ibba, \\ Sergio Lo Caputo, ${ }^{2}$ Francesco Mazzotta, ${ }^{2}$ Daria Trabattoni, and Mario Clerici ${ }^{3,4}$
}

\begin{abstract}
Analyses of immune activation in HIV-exposed seronegative individuals (HESN) yielded discrepant results. To clarify this issue we performed an extensive investigation of immune parameters in HESN and, in particular, we analyzed in these individuals the possible presence of microbial translocation, the most widely accepted reason driving immune activation in HIV-infected patients. Results showed that immune activation, a skewing of T lymphocyte maturation, and increased responsiveness to lipopolysaccharide (LPS) characterize the HESN phenotype; this is not driven by alterations of the gastrointestinal barrier and microbial translocation. The activation state seen in HESN may influence the induction of stronger adaptive antiviral immune responses and may represent a virus exposure-induced innate immune protective phenotype against HIV.
\end{abstract}

$\mathbf{T}$ HE ROLE PLAYED BY IMMUNE ACTIVATION in natural resistance to HIV infection has been largely debated and two different and opposing hypotheses have emerged. The first one holds that HIV-exposed seronegative individuals (HESN) display an immune quiescent phenotype associated with low levels of $\mathrm{CD}^{+}$and $\mathrm{CD}^{+} \mathrm{T}$ lymphocyte activation, reduced $\mathrm{CD}^{+}$and $\mathrm{CD}^{+} \mathrm{T}$ lymphocyte proliferation, and decreased secretion of effector molecules and proinflammatory cytokines. ${ }^{1,2}$ Conversely, data obtained in our and other cohorts indicate that an immune activation phenotype, which is closer to the one in HIV-infected subjects rather than to the one in HIV-uninfected healthy controls (HC), is seen in HESN. ${ }^{3-8}$

Immune activation is a two-edged sword in HIV-1 disease: a negative factor after HIV infection, as it facilitates viral replication and the consequent progression of the disease, ${ }^{9}$ and a possibly favorable factor in controlling susceptibility to the virus, as it raises an immune barrier that could control HIV entry. The study of natural resistance to HIV infection in HESN offers an opportunity to identify mechanisms of "innate" protection that could be exploited in establishing preventive or therapeutic strategies. In an attempt to shed further light on the immune correlates of natural resistance to HIV infection, we investigated whether immune activation is in- deed present in HESN and, if that was the case, if such immune activation would be associated with microbial translocation.

The materials and methods used are detailed in Supplementary Materials and Methods (Supplementary Data are available online at www.liebertpub.com/aid).

Blood samples were collected from 20 Italian HESN, their $\mathrm{HIV}$-infected partners, and from $20 \mathrm{HC}$ enrolled at the Santa Maria Annunziata Hospital in Florence. Inclusion criteria for the HESN were a history of multiple unprotected sexual episodes for more than 4 years at the time of enrollment, with at least three episodes of at-risk intercourse within 4 months prior to study entry, and an average of 30 (range, 18 to >100) reported unprotected sexual contacts per year. Infection in HESN subjects was ruled out by plasma HIV RNA and proviral DNA analyses. None of the subject included in the study was an injectable drug user.

We have been following this cohort of individuals for the past 12 years (the cohort was established in Tuscany at the beginning of 1997). This long period of time has allowed us to adopt very stringent matching criteria between $\mathrm{HC}$ and HESN. Both our HESN and $\mathrm{HC}$ are involved in monogamous relationships, are part of long-lasting couples, and have very similar sexual activities. HC and HESN are from the same

\footnotetext{
${ }^{1}$ Department of Biomedical and Clinical Sciences, University of Milan, Milan, Italy.

${ }^{2}$ S. Maria Annunziata Hospital, Florence, Italy.

${ }^{3}$ Pathophysiology and Transplantation, University of Milan, Milan, Italy.

${ }^{4}$ Don C Gnocchi Foundation, IRCCS, Milan, Italy.
} 
Table 1. Demographic Data of the Enrolled Subjects

\begin{tabular}{|c|c|c|c|}
\hline \multirow[b]{2}{*}{ Characteristics } & \multicolumn{3}{|c|}{ Italy } \\
\hline & $\begin{array}{c}S P \\
(\mathrm{n}=20)\end{array}$ & $\begin{array}{l}H E S N \\
(\mathrm{n}=20)\end{array}$ & $\begin{array}{c}H C \\
(\mathrm{n}=20)\end{array}$ \\
\hline Age, mean years $\pm \mathrm{SD}$ & $41.4 \pm 8.8$ & $40.7 \pm 9.1$ & $42.9 \pm 9.3$ \\
\hline Males, $n(\%)$ & $11(55)$ & $9(45)$ & $10(50)$ \\
\hline Viral load, median copies/ml (range) & $10,250(<50-750,000)$ & $\ldots$ & \\
\hline $\mathrm{CD}^{+} \mathrm{T}$ cell $/ \mu \mathrm{l}$ count, median (range) & $374(36-850)$ & $\ldots$ & \\
\hline $\begin{array}{l}\text { Monthly unprotected sexual episodes, mean } \\
\text { (range) }\end{array}$ & \multicolumn{2}{|c|}{$3(1.5-10)$} & \\
\hline $\begin{array}{l}\text { Previous history of sexually transmitted } \\
\text { diseases and/or AIDS-defining } \\
\text { illnesses }(\%)\end{array}$ & 4 & $\ldots$ & \\
\hline Heterosexual orientation $(\%)$ & 100 & 100 & \\
\hline Ethnicity-ancestry, \% & $\begin{array}{l}\text { European (Tuscan) } \\
\text { ascendency: } 100\end{array}$ & $\begin{array}{l}\text { European (Tuscan) } \\
\text { ascendency: } 100\end{array}$ & $\begin{array}{r}\text { European (Tuscan) } \\
\text { ascendency: } 100\end{array}$ \\
\hline
\end{tabular}

SP, seropositives; HESN, HIV-1-exposed seronegative; HC, healthy controls; SD, standard deviation.

geographic area and share the same genetic background and the same exposure to environmental factors. All individuals (HESN, HIV-1 infected, and HC) had been longitudinally followed for $>4$ years before the study by the Department of Obstetrics and Gynaecology of the S. M. Annunziata Hospital. This allowed us to exclude from the study HESN and HIV-1infected subjects in whom other pathology was reported during the follow-up period. The presence of any chronic disease was an exclusion criteria when the $\mathrm{HC}$ were recruited.

The range of CD4 counts in HIV-1-infected patients was $36-850$ cells $/ \mathrm{ml}$, and viral loads were $>50-750,000$ copies/ $\mathrm{ml}$. All the patients were undergoing antiretroviral (ARV) treatment at the time of the study (Table 1).

The study was designed and performed according to the Helsinki declaration (1975 revised in 2000) and was approved by the Ethics Committee of the participating units. All subjects provided written informed consent to participate in this study.

T lymphocyte activation markers were evaluated by flow cytometry in unstimulated cells. Results showed that the activation profile seen in HESN was closer to the one characterizing HIV-infected subjects than to the one observed in $\mathrm{HC}$. Thus, higher percentages of $\mathrm{CD} 4^{+} / \mathrm{CD} 25^{\text {low }}, \mathrm{CD}^{+} /$
$\mathrm{CD} 8^{+} \mathrm{T}$ lymphocytes, and memory $\mathrm{T}$ cells, as well as lower percentages of naive $T$ cells, were seen in peripheral blood of HESN and HIV-infected individuals compared to HC. Likewise, the expression of $\mathrm{CD} 38^{+} / \mathrm{CD}^{+}$cells was increased in $\mathrm{HESN}$ and $\mathrm{HIV}^{+}$patients compared to $\mathrm{HC}$ even if variances did not reach statistically significant differences (Table 2).

We next investigated the lymphocyte maturation pathway in $\mathrm{HESN}, \mathrm{HC}$, and $\mathrm{HIV}^{+}$patients by analyzing the expression of the chemokine receptor CCR7. Results showed that in HESN patients compared to $\mathrm{HC}$ and HIV patients central memory (CM) $\left(\mathrm{CD} 4^{+} 45 \mathrm{RA}-\mathrm{CCR} 7^{+}\right) \mathrm{CD}^{+}(p<0.01$ and $p<0.009)$ and $\mathrm{CD}^{+}(p<0.05$ and $p<0.04) \mathrm{T}$ cells were augmented, whereas effector memory (EF) $\left(\mathrm{CD}^{+} 45 \mathrm{RA}-\right.$ $\left.\mathrm{CCR}^{-}\right) \mathrm{CD}^{+} \mathrm{T}$ cells were diminished $(p<0.01$ and $p<0.006$ ) (Table 2). Notably, no differences were observed between the three groups of individuals when $\mathrm{CD}^{+} \mathrm{EM}$ cells were analyzed.

Proinflammatory cytokine production was measured by ELISA on lipopolysaccharide (LPS)-stimulated peripheral blood mononuclear cells (PBMCs). Data showed that HESN are more responsive to LPS stimulation than HC. Thus, interleukin (IL)-6 and tumor necrosis factor (TNF)- $\alpha$ levels were significantly higher in HESN compared to $\mathrm{HC}$

Table 2. Percentage \pm SEM of T Cell Markers in Unstimulated Peripheral Blood Mononuclear Cells of Healthy Controls, HIV-Exposed Seronegative Individuals, and HiV ${ }^{+}$SubJects

\begin{tabular}{|c|c|c|c|c|c|c|}
\hline & $H C$ & $H E S N$ & $H I V^{+}$ & $\begin{array}{c}\mathrm{p} \text { value } \\
H C \text { vs HESN }\end{array}$ & $\begin{array}{c}\mathrm{p} \text { value } \\
H C \text { vs } H I V^{+}\end{array}$ & $\begin{array}{c}\mathrm{p} \text { value } \\
H E S N \text { vs } H I V^{+}\end{array}$ \\
\hline $\mathrm{CD}^{+} / 45 \mathrm{RA}^{+} / 62 \mathrm{~L}^{+}$ & $10.6 \pm 2.12$ & $7.98 \pm 2.56$ & $5.7 \pm 1.27$ & 0.06 & 0.01 & ns \\
\hline $\mathrm{CD} 4^{+} / 25^{\text {low }}$ & $8.7 \pm 2.01$ & $17.3 \pm 2.5$ & $7.6 \pm 2.69$ & 0.006 & 0.007 & ns \\
\hline $\mathrm{CD}^{+} / 45 \mathrm{RO}^{+}$ & $29 \pm 4.21$ & $40 \pm 7.8$ & $37 \pm 3.9$ & 0.01 & 0.001 & ns \\
\hline $\mathrm{CD}^{+} / 45 \mathrm{RO}^{+}$ & $1.7 \pm 0.2$ & $10.9 \pm 1.2$ & $6.5 \pm 1$ & 0.008 & 0.039 & ns \\
\hline $\mathrm{CD}^{+} / 38^{+}$ & $9.06 \pm 2.69$ & $20.89 \pm 4.6$ & $23.6 \pm 4.3$ & 0.009 & 0.001 & ns \\
\hline $\mathrm{CD}^{+} / 38^{+}$ & $41 \pm 1.3$ & $51 \pm 2.1$ & $49 \pm 1.21$ & ns & ns & ns \\
\hline $\mathrm{CD}^{+} / 45 \mathrm{RA}^{-} / \mathrm{CCR}^{+}$ & $19.3 \pm 1.2$ & $27.8 \pm 1.2$ & $17.2 \pm 1.3$ & 0.01 & ns & 0.009 \\
\hline $\mathrm{CD}^{+} / 45 \mathrm{RA}^{-} / \mathrm{CCR}^{-}$ & $34.6 \pm 2.6$ & $30.9 \pm 2.4$ & $42.0 \pm 3.6$ & ns & ns & ns \\
\hline $\mathrm{CD}^{+} / 45 \mathrm{RA}^{-} / \mathrm{CCR}^{+}$ & $2.3 \pm 0.3$ & $18.5 \pm 2.6$ & $5.4 \pm 0.8$ & 0.05 & ns & 0.04 \\
\hline $\mathrm{CD}^{+} / 45 \mathrm{RA}^{-} / \mathrm{CCR}^{-}$ & $33.2 \pm 2.4$ & $16.4 \pm 2$ & $42 \pm 3.4$ & 0.01 & ns & 0.006 \\
\hline
\end{tabular}

ns, nonsignificant.

Statistical analyses: Mann-Whitney $U$ test. 


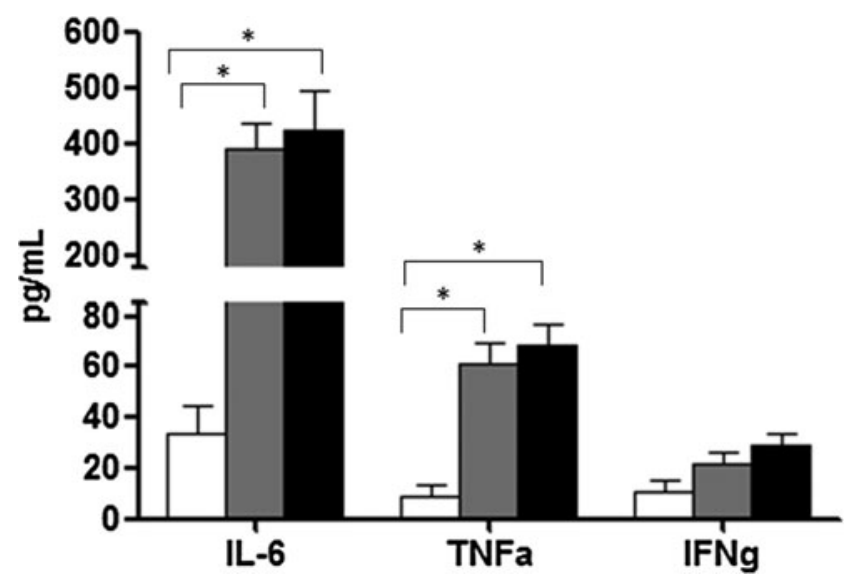

FIG. 1. Cytokine production in lipopolysaccharide (LPS)stimulated peripheral blood mononuclear cells (PBMCs). Interleukin (IL)-6, tumor necrosis factor (TNF)- $\alpha$, and interferon (IFN)- $\gamma$ production by LPS-stimulated PBMCs of HIV-exposed seronegative individuals (HESN), healthy controls (HC), and $\mathrm{HIV}^{+}$individuals; results are shown as fold-change expression from the unstimulated sample.

$(p<0.05)$, and were similar to those observed in HIV patients. Interferon (IFN)- $\gamma$ showed a similar trend, although differences did not reach statistical differences (Fig. 1).

To determine whether the increase in LPS-stimulated production of proinflammatory cytokines seen in HESN was associated with an augmented expression of TLR on immune cells this parameter was analyzed on unstimulated and LPSstimulated $\mathrm{CD}_{1} 4^{+}$cells. No differences emerged in any of the conditions either in the percentage of $\mathrm{TLR} 4^{+} / \mathrm{CD} 14^{+}$cells or in mean fluorescence intensity (MFI) (data not shown).

The expression of proteins that are part of the TLR signal transduction pathway was analyzed next using a real time polymerase chain reaction (PCR) array. Results showed that higher mRNA levels of a number of such proteins, including CSF2, CSF3, IFN- $\beta$, IL-10, IL-12, IL-1 $\alpha$, IL-1 $\beta$, IL-2, IL-6, $\mathrm{COX} 2$, and TNF- $\alpha$, were present in HESN compared to HC (Supplementary Fig. S1) Notably, results obtained in HESN were similar to those seen in HIV patients. These data were further validated by real-time PCR on individual samples (Fig. 2).

LPS plasma level was measured next to determine if the inflammatory phenotype seen in HESN could be justified by an alteration of this parameter analogous to what has been shown in HIV patients. Results showed that the concentration of plasma LPS in HESN was significantly lower than the one observed in HIV patients, and was similar to what was detected in HC (Fig. 3A). Similar results were observed when plasma levels of soluble CD14 (sCD14) were analyzed, suggesting that exposure to HIV in the absence of infection does not result in alterations of the gastrointestinal barrier in HESN (Fig. 3B).

The early stages of HIV-1 infection are characterized by massive damage that affects the intestinal mucosa and results in microbial translocation from the intestinal lumen into the systemic circulation. ${ }^{8}$ This results in increased plasma levels of LPS, a component of the Gram-negative bacterial cell membrane; upon interacting with LPS binding protein (LBP), LPS promotes the release of $\mathrm{sCD} 14$ by monocyte/macrophages. ${ }^{10}$ Notably, LPS binding of Toll-like receptor-4 (TLR4) expressed on different cell types elicits a widespread systemic immune activation and inflammation. ${ }^{11,12}$

Based on these premises we wondered if the immune activation state we observe in HESN could result in activationinduced cell death and consequent microbial translocation. To this end we evaluated LPS and sCD14 plasma levels. Results showed that these markers have similar concentrations in HESN and HC, thus excluding the possibility of establishing a correlation between immune activation and microbial translocation in these subjects. Analysis of the expression of genes involved in TLR signaling nevertheless showed that upon LPS stimulation, a number of effector molecules (CSF2, CSF3, IFN- $\beta$, IL-10, IL-12, IL-1 $\alpha$, IL-1 $\beta$, IL-2, IL-6, COX2, and TN-F $\alpha$ ) were upregulated in $\mathrm{HIV}^{+}$ individuals and HESN.

Similarly, the production of proinflammatory cytokines in response to TLR triggering was increased in HESN and was comparable to that of HIV patients. Importantly, the increased responsiveness of TLR in HESN and HIV-infected

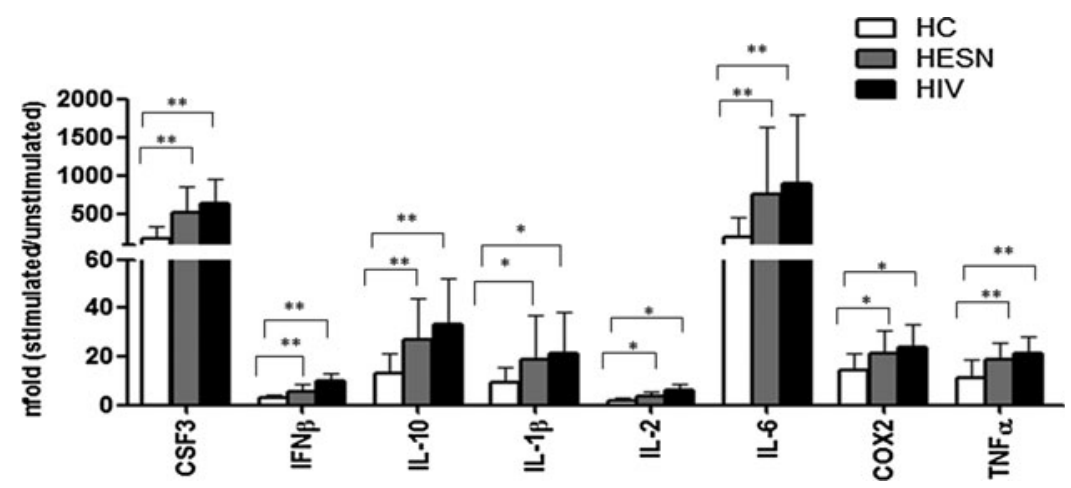

FIG. 2. Effector molecules of the toll-like receptor (TLR) signaling pathway analyzed by individual real time polymerase chain reaction (PCR). Those targets showing marked differences between HESN, $\mathrm{HIV}^{+}$, and HC in the PCR array were retested by real-time PCR on individual samples. CSF3, IFN- $\beta$, IL-10, IL-1 $\beta$, IL-2, IL-6, COX2, and TNF- $\alpha$ expression was assessed by real-time quantitative RT-PCR after stimulation of HC (white bars), HESN (gray bars), and HIV ${ }^{+}$(black bars) PBMCs with LPS (TLR4) for $6 \mathrm{~h}$, calculated relative to a housekeeping gene and shown as fold-change expression from the unstimulated sample. ${ }^{*} p<0.05 ; * * p<0.01$. 
A

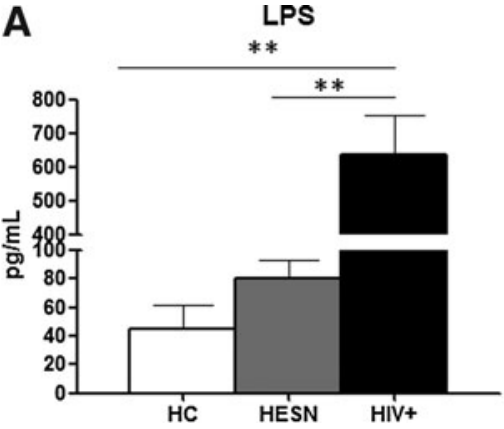

B

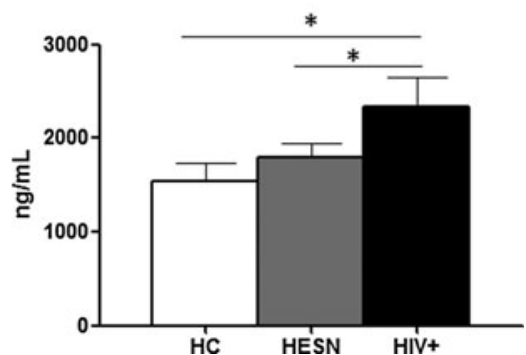

FIG. 3. LPS and sCD14 plasma concentration. Plasma concentrations of (A) LPS and (B) of sCD14 in HC (white bars), HESN (gray bars), and $\mathrm{HIV}^{+}$individuals (black bars). $* p<0.05 ; * * p<0.01$. individuals was not associated with changes in TLR expression. The increased TLR4 sensitization in our HESN cohort is therefore dependent on mechanisms other than increased TLR4 expression. Periodic exposure to HIV through unprotected sexual intercourse or a peculiar genetic predisposition could possibly justify this phenomenon and should be further explored.

These results are in contrast to previously reported data suggesting lower expression of proinflammatory cytokines in association to the HESN phenotype at both the mucosal and systemic level. ${ }^{13-15}$ Yao et al. ${ }^{16}$ in particular reported diminished TLR4 expression and similar responsiveness to TLR4 stimulation in cervical mononuclear cells (CMC) of their HESN cohort. However, differences in ethnicity, biological sample (CMC and PBMCs), and modalities of exposure (occasional and massive) to HIV in the Italian and Nairobi cohorts, respectively, could at least partially justify these apparent inconsistencies.

Actually, the presence of higher levels of proinflammatory cytokines (such as IL-6, TNF- $\alpha$, and IL-1) in HESN is apparently difficult to justify because different reports suggest that $\mathrm{T}$ cell activation facilitates the spread of HIV-1 infection. ${ }^{17,18}$ Nevertheless, the systemic immune activation, together with the higher percentage of central memory $\mathrm{T}$ cells that we found was associated with the HESN phenotype, is biologically interesting. Indeed, it is tempting to picture a situation in which, following viral exposure, HESN would respond promptly by triggering an effector molecule storm capable of blocking viral replication and impeding viral diffusion, thus lowering the chance of overt infection. Furthermore, immune activation could influence the induction of stronger adaptive antiviral immune responses and may represent a virus exposure-induced innate immune protective phenotype against HIV.

Immunological analyses performed in HESN have shown conflicting results. Thus, whether immune quiescence has been repeatedly observed to be present in the "Pumwani cohort" of Kenyan sexual workers, ${ }^{1,2}$ immune activation was described in our and other cohorts of HESN. ${ }^{3-8}$ Results herein indicate that at least in some cohorts, resistance to HIV infection is associated with immune activation. A likely working hypothesis to justify these differences is that they are a consequence of the antigenic load: extremely high and repeated exposure to HIV in African sex workers and much more sporadic contact with the virus in our and other groups of HESN in whom immune activation is observed. This and other possibilities will need to be further investigated.

\section{Acknowledgments}

HIV-1Ba-L was provided through the EU program EVA center for AIDS Reagents NIBSC, UK. We thank the healthy blood donors at Santa Maria Annunziata Hospital in Florence included in the study as healthy controls.

\section{Author Disclosure Statement}

No competing financial interests exist.

\section{References}

1. Card CM, Ball TB, and Fowke KR: Immuno quiescence: A model of protection against HIV-1 infection. Retrovirology 2013;20;10(1):141.

2. McLaren P, Ball T, Wachihi C, et al.: HIV-exposed seronegative commercial sex workers show a quiescent phenotype in the CD4+ $\mathrm{T}$ cell compartment and reduced expression of HIV-dependent host factors. J Infect Dis 2010;202:S339-S344.

3. Miyazawa M, Lopalco L, Mazzotta F, et al.: The "immunological advantage" of HIV-exposed seronegative individuals. AIDS 2009;14;23(2):161-175.

4. Biasin M, Caputo SL, Speciale L, et al.: Mucosal and systemic immune activation is present in human immunodeficiency virus-exposed seronegative women. J Infect Dis 2000;182(5):1365-1374.

5. Biasin M, Piacentini L, Lo Caputo S, et al.: TLR activation pathways in HIV-1-exposed seronegative individuals. J Immunol 2010;184(5):2710-2717.

6. Jennes W, Sawadogo S, Koblavi-Deme S, et al.: Cellular human immunodeficiency virus (HIV)-protective factors: A comparison of HIV-exposed seronegative female sex workers and female blood donors in Abidjan, Cote d'Ivoire. J Infect Dis 2003;187(4):206-214.

7. Tran HK, Chartier L, Troung LX, et al.: Systemic immune activation in HIV-1-exposed uninfected Vietnamese intravascular drug users. AIDS Res Hum Retroviruses 2006; 22:255-261.

8. Suy A, Castro P, Nomdedeu M, et al.: Immunological profile of heterosexual highly HIV-exposed uninfected individuals: predominant role of CD4 and CD8 T-cell activation. J Infect Dis 2007;196(6):1191-1201.

9. Mehandru S, Poles MA, Tenner-Racz K, et al.: Primary HIV-1 infection is associated with preferential depletion of $\mathrm{CD}^{+} \mathrm{T}$ lymphocytes from effector sites in the gastrointestinal tract. J Exp Med 2004;200(6):761-770.

10. Neaton JD, Neuhaus J, and Emery S: Soluble biomarkers and morbidity and mortality among people infected with 
HIV: Summary of published reports from 1997 to 2010. Curr Opin HIV AIDS 2010;5:480-490.

11. Jiang W, Poles MA, Tenner-Racz K, et al.: Plasma levels of bacterial DNA correlate with immune activation and the magnitude of immune restoration in persons with antiretroviral-treated HIV infection. J Infect Dis 2009; 199(8): 1177-1185.

12. Shan L and Siliciano RF: Unraveling the relationship between microbial translocation and systemic immune activation in HIV infection. J Clin Invest 2014;124(6): 2368-2371.

13. Lajoie J, Kimani M, Plummer FA, et al.: Association of sex work with reduced activation of the mucosal immune system. Infect Dis 2014;210(2):319-329.

14. Prodger JL, Hirbod T, Kigozi G, et al.: Immune correlates of HIV exposure without infection in foreskins of men from Rakai, Uganda. Mucosal Immunol 2014;7(3):634-644.

15. Taborda NA, Hernández JC, Lajoie J, et al.: Low expression of activation and inhibitory molecules on NK cells and CD4+ $\mathrm{T}$ cells is associated with viral control. AIDS Res Hum Retroviruses 2015;31(6):636-640.

16. Yao XD, Omange RW, Henrick BM, et al.: Acting locally: Innate mucosal immunity in resistance to HIV-1 infection in Kenyan commercial sex workers. Mucosal Immunol 2014;7(2):268-279.

17. Naranbhai V, Abdool Karim SS, Altfeld M, et al.: Innate immune activation enhances HIV acquisition in women, diminishing the effectiveness of tenofovir microbicide gel. J Infect Dis 2012;206:993-1001.

18. Kahle EM, Bolton M, and Hughes JP: Plasma cytokine levels and risk of HIV Type 1 (HIV-1). Transmission and acquisition: A nested case-control study among HIV-1serodiscordant couples. J Infect Dis 2015;211(9):14511460 .

Address correspondence to:

Mara Biasin

Chair of Immunology University of Milan

Department of Biomedical and Clinical Sciences "L. Sacco",

Via G.B. Grassi 74 20157 Milan Italy

E-mail: mara.biasin@unimi.it 